\title{
Anticipatory Cognitive Stress Appraisal and the Acute Procoagulant Stress Response in Men
}

Petra H. Wirtz, PhD, Ulrike Ehlert, PhD, Luljeta Emini, MS, Katharina Rüdisüli, MS, Sara Groessbauer, MS, Jens GaAb, PhD, Sigrid Elsenbruch, PhD, and Roland von Känel, MD

\begin{abstract}
Objective: Acute mental stress elicits blood hypercoagulability. Following a transactional stress model, we investigated whether individuals who anticipate stress as more threatening, challenging, and as exceeding their coping skills show greater stress reactivity of the coagulation activation marker D-dimer, indicating fibrin generation in plasma. Methods: Forty-seven men (mean age $44 \pm$ 14 years; mean blood pressure [MBP] $101 \pm 12 \mathrm{~mm} \mathrm{Hg}$; mean body mass index [BMI] $26 \pm 3 \mathrm{~kg} / \mathrm{m}^{2}$ ) completed the Primary Appraisal Secondary Appraisal (PASA) scale before undergoing the Trier Social Stress Test (combination of mock job interview and mental arithmetic task). Heart rate, blood pressure, plasma catecholamines, and D-dimer levels were measured before and after stress, and during recovery up to 60 minutes poststress. Results: Hemodynamic measures, catecholamines, and D-dimer changed across all time points ( $p$ values <.001). The PASA "Stress Index" (integrated measure of transactional stress perception) correlated with total D-dimer area under the curve (AUC) between rest and 60 minutes poststress $(r=0.30, p=.050)$ and with D-dimer change from rest to immediately poststress $(r=0.29, p=.046)$. Primary appraisal (combined "threat" and "challenge") correlated with total D-dimer AUC $(r=0.37, p=.017)$, D-dimer stress change $(r=0.41, p=.004)$, and $\mathrm{D}$-dimer recovery $(r=0.32, p=$ $.042)$. "Challenge" correlated more strongly with D-dimer stress change than "threat" $(p=.020)$. Primary appraisal $\left(\Delta \mathrm{R}^{2}=0.098\right.$, $\beta=0.37, p=.019)$, and particularly its subscale "challenge" $\left(\Delta \mathrm{R}^{2}=0.138, \beta=0.40, p=.005\right)$, predicted $\mathrm{D}$-dimer stress change independently of age, BP, BMI, and catecholamine change. Conclusions: Anticipatory cognitive appraisal determined the extent of coagulation activation to and recovery from stress in men. Particularly individuals who anticipated the stressor as more challenging and also more threatening had a greater fibrin stress response. Key words: blood coagulation, cognitive appraisal, psychological stress.
\end{abstract}

$\mathbf{A U C}=$ area under the curve; $\mathbf{B M I}=$ body mass index; $\mathbf{C A D}=$ coronary artery disease; $\mathbf{E P I}=$ epinephrine; $\mathbf{H R}=$ heart rate; $\mathbf{M B P}=$ mean blood pressure; NEPI = norepinephrine; PASA = Primary Appraisal Secondary Appraisal; TSST $=$ Trier Social Stress Test.

\section{INTRODUCTION}

$\mathrm{T}$ he role of the hemostatic system is to maintain blood in a fluid state under physiological conditions, thereby preventing the organism from excessive bleeding on the one hand and thrombus formation on the other (1). A growing body of research has convincingly demonstrated that in healthy individuals, acute mental stress concomitantly activates both the coagulation and the fibrinolysis part of hemostasis to result in net blood hypercoagulability (2). As early as 1914, Cannon stated that "rapid coagulation may reasonably be considered as an instance of adaptive reaction serviceable to the organism in the injury which may follow the struggle that fear or rage may occasion" (3). The thickening of blood in response to an acute and potentially life-threatening stressor is therefore physiological, because it provided our ancestors with an evolutionary benefit (2). Under certain circumstances, however, a procoagulant stress response may incur harm for the cardio-

From the Department of Clinical Psychology and Psychotherapy, University of Zurich, Zurich, Switzerland (P.H.W., U.E., L.E., K.R., S.G., J.G.); the Department of Medical Psychology, University Hospital of Essen Medical School, Essen, Germany (S.E.); the Department of General Internal Medicine, University Hospital Berne, Berne, Switzerland (R.v.K.); and the Psychocardiology Unit, Cardiovascular Prevention and Rehabilitation, Swiss Cardiovascular Center, University Hospital Berne, Berne, Switzerland (R.v.K.).

Address correspondence and reprint requests to Roland von Känel, MD, Professor of Medicine and Head of Psychosomatic Division, Department of General Internal Medicine University Hospital/INSELSPITAL, CH-3010 Bern, Switzerland. E-mail: roland.vonkaenel@insel.ch

Received for pulication January 26, 2006; revision received July 11, 2006.

This work was supported by research grant 2003 from the University of Zurich, Switzerland (to P.H.W.) and by a research grant from the University of Berne, Switzerland (to R.v.K.). vascular system because hypercoagulability could contribute to overt thrombosis (4). For instance, individuals with preexisting atherosclerotic diseases exhibit exaggerated hypercoagulability in response to acute stress (5-8). If, in patients with coronary artery disease (CAD), a stress-induced blood pressure peak provokes rupture of a vulnerable atherosclerotic plaque (9), a hypercoagulable milieu could accelerate intracoronary thrombus growth and critical vessel occlusion (4). Therefore, a better insight into factors that might exaggerate the procoagulant stress response to an extent that may become harmful for certain populations could have clinical value.

Aside from manifest cardiovascular diseases (5-8), we have previously identified several psychosocial factors that predict procoagulant stress reactivity. Elderly subjects who indicated relatively more life stress in the preceding weeks (10), those with relatively more anxiety symptoms (11), and those showing relatively lower levels of approach coping (12) had a greater increase in the procoagulant stress response to a speech task. The latter study in particular suggested that poor coping strategies could contribute to increased coagulation activation with acute stress, in this case impaired competence in coping with a contextual challenge (13). This observation is potentially important because it provides clinicians with the possibility to intervene in the case of high-risk cardiovascular patients who cope poorly with stress.

We therefore aimed to further elucidate the tentative notion that coping processes are related to procoagulant reactivity to stress and restoration of hypercoagulability during recovery from stress. The concept of coping with stress and its relationship to cardiovascular reactivity has produced a wealth of data. However, as yet, the previous studies do not allow any firm conclusions to be drawn regarding which concept should be favored over others as a result of the large number of different coping classifications used across the different studies (14). One conclusion from this research is that reactivity 
studies commonly applied questionnaires that investigate coping skills that may have little value for effective coping with the actual stress experiment. We therefore decided to apply the transactional stress model as proposed by Lazarus and Folkman (15). This theory posits that people's interpretations of an event, their personal resources, and characteristics of the stressor interact to determine the subjective meaning of the stressor, people's coping efforts, and, importantly, also their psychophysiological reactions (16). The model further allows for an assessment of anticipatory cognitive appraisal of a pending stressor, a process that showed a higher correlation with psychophysiological stress responses than retrospective appraisal of a previous stressor (17).

From the many hemostatic molecules (2), we chose to measure the coagulation activation marker D-dimer because, as opposed to individual clotting factors of blood coagulation pathways, D-dimer indicates activation of the entire coagulation and fibrinolysis systems (18). More precisely, D-dimer is generated when the fibrinolytic enzyme plasmin dissolves fibrin, which is the end product downstream in the coagulation cascade and the main component of a blood clot (19). D-dimer shows reliable responsiveness to acute stress and is perhaps more readily detected in the plasma of stressed individuals than other hemostasis molecules as a result of its comparably long half-life (18). Research suggests that D-dimer is cleared from the blood with an average in vivo half-life of approximately 7 hours (20). However, the molar concentration of D-dimer is regulated by several processes, including plasmin generation and inhibition of plasmin by antiplasmin, plasmin degradation of fibrin forming D-dimers, and hepatic clearance of D-dimer (20), all of which may vary across different study protocols. In a sample of middle-aged healthy men different from those investigated here (21), we previously found that D-dimer had returned to prestress resting levels 45 minutes after stress termination. This kinetic suggests that D-dimer is a suitable marker for protocols in which the acute hemostatic stress response is assessed during a recovery period of up to 1 hour after stress termination. The clinical use of D-dimer is highlighted by its value as a sensitive marker of a thrombogenic state (19) that is routinely measured in the diagnostic workup of a suspected venous thromboembolic event (22). Age, blood pressure (BP), and body mass index (BMI) are common covariates of resting D-dimer levels (23-26). Nonetheless, a recent meta-analysis established the role of plasma D-dimer as a significant and independent predictor of firsttime and recurrent cardiovascular events over a median follow up of 5 years (23).

We hypothesized that men who anticipate the stressor as more threatening and challenging, and expect themselves to have less competence and control in coping with the stressor, would show greater stress responsiveness and prolonged stress recovery of D-dimer. We further hypothesized that stress appraisal would predict stress-induced D-dimer change in part independently of the usual covariates and also independently of catecholamine surge previously shown to elicit hypercoagulability (27). An exaggerated acute physiological stress response and delayed restoration of perturbed physiology after stress is shut off are both important in understanding the adverse effects of stress on physical health (28). We therefore did not restrict our analyses to the D-dimer change over the entire stress and recovery interval, but also investigated the relationship between stress appraisal and the acute stress change in D-dimer and its recovery from stress separately. Because the female cycle affects resting coagulant function (29) and coagulation responses to stress (30), we did not include women in the present study.

\section{MATERIALS AND METHODS Study Participants}

The study protocol was formally approved by the Ethics Committee of the State of Zurich, Switzerland. All subjects provided written informed consent to a protocol studying different aspects of psychophysiological stress reactivity. The final study sample comprised 47 men who varied in age (range, 20-65 years), screening systolic (range, 108-167 $\mathrm{mm} \mathrm{Hg}$ ) and diastolic (range, 65-114 mm Hg) BP, and BMI (range, 20-35 kg/m²). Recruitment was carried out through advertisement of the study on pin boards at the University of Zurich and by members of the research team who accompanied the mobile blood donation unit of the Swiss Red Cross of the State of Zurich. The study was conducted between April and October 2004.

All participants were required to be nonsmokers, to not take any regular or occasional medication, and to be in excellent physical and mental health as confirmed by an extensive health questionnaire (31) and telephone interview. Specific exclusion criteria were self-report of regular heavy physical exercise, alcohol and illicit drug abuse, any heart disease, varicosis or thrombotic diseases, elevated blood sugar and diabetes, elevated cholesterol, liver and renal diseases, chronic obstructive pulmonary disease, allergies and atopic diathesis, rheumatic diseases, and current infectious diseases. The general practitioner was contacted if the personal or medication history was inconclusive.

All eligible subjects received three seated BP assessments after a 15minute rest using a fully automated sphygmomanometry device (Omron 773; Omron Healthcare Europe B.V. Hoofddorp, The Netherlands) on 3 different days. The average mean arterial BP (MBP) was calculated by the formula $(2 / 3$ * diastolic BP $)+(1 / 3 *$ systolic BP) and defined as screening BP. BMI was calculated as the ratio of weight in kilograms to height in square meters.

\section{Stress Protocol}

Subjects were tested between 2:00 PM and 4:00 PM after having abstained from physical exercise, alcohol, and caffeinated beverages since the previous evening. We applied the standard protocol of the widely used Trier Social Stress Test (TSST) as introduced by Kirschbaum et al. (32). The TSST combines a short introduction that is followed by a 5-minute preparation phase, a 5-minute mock job interview, and a 5-minute mental arithmetic task in this sequence before an audience (32). All subjects completed the selfreport Primary Appraisal Secondary Appraisal (PASA) scale (see subsequently) at the end of the 5-minute preparation phase, immediately before the beginning of the mock job interview. During the recovery phase, subjects remained seated in a quiet room for 60 minutes.

Heart rate (HR) was continuously assessed every minute by a portable HR monitor (Polar S810; Polar Electro, Kempele, Finland). Mean HR responses were measured and averaged across the 5-minute immediate prestress period, across the 10-minute stressor (5-minute speech task and 5-minute mental arithmetic combined), and 20 minutes after stress termination (33). BP was measured every 15 beats from the radial pulse waveform using the Vasotrac APM205A device (Medwave Inc., St. Paul, MN). BP values were averaged for the 5-minute interval immediately before stress, across the job interview and arithmetic task combined, and across 16 to 20 minutes poststress. BP measured noninvasively by the Vasotrac previously demonstrated excellent correlation with BP measured through a radial arterial catheter, and the mean difference in MBP measured by the Vasotrac versus the radial arterial catheter 
was $<3 \mathrm{~mm} \mathrm{Hg}$ (34). However, Vasotrac readings overestimate sphygmomanometric BP readings (35). We therefore present BP data for the sole purpose of demonstrating that the TSST elicited a significant change in BP.

\section{Transactional Stress Questionnaire}

We applied a transactional stress questionnaire - the PASA scale — which we recently developed in the German language in a sample of male nonsmoking students without any acute or chronic somatic or psychiatric disorders (17). The PASA questionnaire was designed to fit with the respective description of the transactional stress model proposed by Lazarus and Folkman, which emphasizes that the characteristics of the person and the stressor interact to determine the subjective meaning of the stressor (15). The specific use of the questionnaire for stress research is the specific relation of its items to the anticipated cognitive appraisal of the TSST.

In brief, the PASA assesses four anticipatory cognitive appraisal processes, namely "threat" (e.g., I do not feel threatened by the situation), "challenge" (e.g., the situation is not a challenge for me), "self-concept of own abilities" (e.g., it mainly depends on me whether the experts judge me positively), and "control expectancy" (e.g., I have no idea what I should do now). Each of these primary PASA scales comprises four items that are rated on a 6-point Likert scale ranging from "strongly disagree" to "strongly agree." Primary PASA scales are combined into two secondary PASA scales, namely in a "primary appraisal" scale, which comprises the items "threat" and "challenge," and in a "secondary appraisal" scale, which comprises the items "self-concept" and "control expectancy." Primary appraisal refers to a person's judgment about the significance of an event as stressful, positive, controllable, challenging, or irrelevant, whereas secondary appraisal assesses available coping resources and options when faced with a stressor (17). Eventually, a "tertiary appraisal," or global PASA scale, termed "stress index," is computed, which combines "primary appraisal" and "secondary appraisal" scales to provide an integrated measure of transactional stress perception that is calculated by the following formula: "threat" + "challenge" "self-concept" - "control expectancy" (17). The specific purpose of "tertiary appraisal" is to provide a construct that meets Lazarus and Folkman's paradigm of the mutual dependency of primary and secondary processes in anticipated cognitive stress appraisal (15). Thus, a higher "tertiary appraisal" score represents a higher general stress perception.

Our recent validation of the PASA questionnaire yielded a four-factor solution (i.e., the four primary PASA scales), which was considered optimal for the data set and explained a total of $62 \%$ of the variance after rotation. Cronbach's alpha was calculated with primary PASA scales showing reasonable $(>0.60)$ to good $(>0.80)$ internal consistency ("threat": $\alpha=0.83$; "challenge": $\alpha=0.63$; "self-concept of own competence": $\alpha=0.81$; "control expectancy": $\alpha=0.77$ ) (17). Further factor analysis on the basis of the primary scales "threat," "challenge," "self-concept of own competence," and "control expectancy" resulted in two factors with the primary scales "threat" and "challenge" (factor 1 labeled "primary appraisal") and "self-concept of own competence" and "control expectancy" (factor 2 labeled "secondary appraisal"). These secondary scales explained a total variance of $73 \%$ and showed good ("primary appraisal": $\alpha=0.80$ ) or reasonable ("secondary appraisal": $\alpha=0.74$ ) internal consistency (17).

\section{Biochemical Measures}

Venous blood for D-dimer measures was drawn through an indwelling 18-gauge forearm catheter (Venflon, BD, Basel, Switzerland) from the nondominant arm into polypropylene tubes (Sarstedt, Sevelen, Switzerland) containing 3.8\% sodium citrate $(9: 1 \mathrm{v} / \mathrm{v})$. Samples were centrifuged at $2000 \mathrm{~g}$ for 20 minutes at $4^{\circ} \mathrm{C}$. For catecholamine assays, venous blood was drawn into EDTA-coated monovettes (Sarstedt, Numbrecht, Germany) and centrifuged for 10 minutes at $2000 \mathrm{~g}$ at $4^{\circ} \mathrm{C}$. All plasma samples were immediately stored at $-80^{\circ} \mathrm{C}$ until further analysis. D-dimer was determined by an enzymelinked immunosorbent assay (Asserachrom Stago, Asnières, France), and norepinephrine (NEPI) and epinephrine (EPI) levels were determined by means of high-performance liquid chromatography and electrochemical detection after liquid-liquid extraction (36). Inter- and intraassay coefficients of variation were $<10 \%$ for $\mathrm{D}$-dimer and catecholamine analyses.
Blood for D-dimer and catecholamine assays was obtained immediately before the beginning of the TSST, immediately after stress, and 20 minutes and 60 minutes after stress. For catecholamine measures, an additional blood sample was drawn 10 minutes poststress.

\section{Statistical Analyses}

Data were analyzed using SPSS statistical software package version 12.0 (SPSS Inc., Chicago, IL). All testing was two-tailed with the significance level set at $p \leq .05$. Normal distribution of data was verified by the Kolmogorov-Smirnov test. BMI values were logarithmically transformed. Pearson correlation analysis was used to estimate the bivariate relationship between two continuous variables. Analysis of variance for repeated measures was computed to investigate whether the stressor provoked a significant change in biological systems across various time points. Post hoc analyses applied Fisher least significant difference. Degrees of freedom vary across analyses because data were occasionally missing as a result of technical problems; i.e., D-dimer levels and EPI levels 60 minutes poststress were missing in five and three subjects, respectively; EPI levels 10 and 20 minutes poststress were each missing in one subject.

We used a previously published formula to compute the area under the curve (AUC) with respect to increase for D-dimer and catecholamine levels across time intervals (37). The formula considers the variable time between measurements and ignores the distance from zero for all measurements, thereby emphasizing a variable's integrated change over time. D-dimer and catecholamine AUC measures were computed between rest and 60 minutes after stress ("total AUC"), between rest and immediately poststress ("stress change"), and between immediately poststress and 60 minutes thereafter ("recovery AUC"). To avoid spurious associations because of multiple comparisons, only if D-dimer total AUC was significantly associated with a PASA scale were further analyses computed to identify post hoc whether the scale correlated with D-dimer stress change, D-dimer recovery AUC, or both. As a result of a nonnormal distribution, D-dimer total AUC and D-dimer recovery AUC measures were subject to Blom normal score transformation before statistical procedures were performed.

We applied hierarchical linear regression analysis to investigate whether PASA scales are independent predictors of D-dimer measures. According to common statistical recommendations for the ratio of observations to predictors, our sample size allowed for regression equations that considered a maximum of five predictors (38). In addition to one of the PASA scales, we therefore defined a priori four potential confounders of the relationship between PASA scales and D-dimer measures. These were previously established covariates of resting D-dimer levels and D-dimer stress reactivity, namely age, BMI, screening BP, and catecholamine activity (23-27). We eliminated redundant predictors by combining screening systolic BP and diastolic BP into screening MBP, and prioritized NEPI over EPI measures because NEPI data were complete in all subjects. In the final models, age, BMI, screening MBP, NEPI activity, and one of the PASA scales were entered as predictors and regressed on D-dimer measures in this order.

\section{RESULTS \\ Subjects' Characteristics}

Table 1 presents the biological and psychological characteristics of the 47 men studied. As intended, some subjects had mild screening hypertension (systolic BP $\geq 140 \mathrm{~mm} \mathrm{Hg}$ and/or diastolic $\mathrm{BP} \geq 90 \mathrm{~mm} \mathrm{Hg} ; n=19)$ and were obese (BMI $\geq 30$ $\left.\mathrm{kg} / \mathrm{m}^{2} ; n=4\right)$. Intercorrelations between PASA primary, secondary, and tertiary scales were comparable with our previous observations (17).

\section{Stress Reactivity}

Mean \pm standard error of mean HR $(78.2 \pm 1.8,89.0 \pm$ $2.6,72.6 \pm 1.5$ beats $\left./ \mathrm{min} ; \mathrm{F}_{2,45}=50.7, p<.001\right)$, systolic BP $\left(180 \pm 4.4,194 \pm 3.9,163 \pm 2.8 \mathrm{~mm} \mathrm{Hg} \mathrm{F}_{2,39}=31.4, p<\right.$ $.001)$, and diastolic BP $(104 \pm 2.4,117 \pm 2.9,99 \pm 2.0 \mathrm{~mm}$ 
$\left.\mathrm{Hg} ; \mathrm{F}_{2,39}=22.0, p<.001\right)$ significantly changed between rest, immediately poststress, and recovery from stress. Figure 1 shows that NEPI $\left(\mathrm{F}_{4,43}=46.7, p<.001\right.$; Fig. 1A), EPI $\left(\mathrm{F}_{4,38}=9.0, p<.001\right.$; Fig. 1B $)$, and D-dimer $\left(\mathrm{F}_{3,39}=8.3\right.$, $p<.001$; Fig. 1C) did also significantly change between rest, poststress, and recovery.

In post hoc analyses, increase in hemodynamic measures, catecholamines, and D-dimer were all significant between rest and immediately poststress (all $p$ values $\leq .001$ ), demonstrating that the stressor provoked a reliable response in all biological systems studied. In terms of recovery, $\operatorname{HR}(p<.001)$ and systolic BP $(p<.001)$ were significantly lower 20 minutes after stress compared with rest, whereas diastolic BP had returned to resting values. EPI and D-dimer levels had both returned to resting values 60 minutes after stress, and NEPI levels were higher 60 minutes after stress compared with rest $(p<.001)$.

\section{Primary Appraisal Secondary Appraisal Scales and D-dimer Measures}

Table 2 summarizes the analyses for the crude relationships among PASA primary, secondary, and tertiary scales and D-dimer measures for the different time points and intervals.

TABLE 1. Biological and Psychological Characteristics of the 47 Subjects Studied

\begin{tabular}{lc}
\hline Age [years] & $43.7 \pm 13.6(21$ to 65$)$ \\
Body mass index $\left[\mathrm{kg} / \mathrm{m}^{2}\right]$ & $25.8 \pm 2.9(20.7$ to 34.3$)$ \\
Mean blood pressure $[\mathrm{mm} \mathrm{Hg}]$ & $101.3 \pm 12.4(82.8$ to 131.2$)$ \\
PASA threat & $4.3 \pm 1.1(1.8$ to 6.0$)$ \\
PASA challenge & $3.0 \pm 0.9(1.5$ to 5.3$)$ \\
PASA primary appraisal & $3.7 \pm 0.8(2.0$ to 5.6$)$ \\
PASA self-concept & $2.8 \pm 0.9(1.3$ to 5.3$)$ \\
PASA control expectancy & $2.4 \pm 0.8(1.0$ to 4.5$)$ \\
PASA secondary appraisal & $2.6 \pm 0.7(1.4$ to 4.0$)$ \\
PASA stress index & $2.2 \pm 2.5(-3.0$ to 6.8$)$ \\
\hline
\end{tabular}

Values are given as means \pm standard deviation (range). PASA $=$ Primary Appraisal Secondary Appraisal scale.

\section{Omnibus Test}

As hypothesized, an omnibus test showed that the tertiary PASA scale "stress index" was significantly associated with D-dimer total AUC across the four time points assessed ( $r=$ $0.30, p=.050$; Fig. 2A) but was not associated with resting D-dimer levels $(p=.68)$. This observation suggested that the entire process of cognitive appraisal was specifically related to D-dimer change elicited by the stressor. Moreover, as presented in Table 2, post hoc analysis suggests that "stress index" was significantly related to acute change in D-dimer from rest to immediately poststress (i.e., D-dimer stress change) ( $r=0.29, p=.046)$. "Stress index" also showed a trend toward statistical significance for a correlation with the integrated change in recovery of D-dimer levels between immediately poststress and 60 minutes thereafter (i.e., D-dimer recovery AUC) $(r=0.27, p=.087)$.

\section{PASA Primary and Secondary Appraisal}

The significance of the omnibus test justified a specific analysis in terms of whether "primary appraisal," "secondary appraisal," or both would be significantly related to change in total D-dimer AUC. To account for multiple comparisons, we required a significance level of $p=.025$ for this analysis (i.e., $p=.05 / 2$ for "primary appraisal" and "secondary appraisal"). Table 2 shows that "primary appraisal" $(p=.017)$ but not "secondary appraisal" ( $p=.46)$ was significantly associated with D-dimer total AUC. In post hoc analysis, "primary appraisal" correlated with both D-dimer stress change $(p=.004)$ and D-dimer recovery AUC $(p=.042)$.

\section{PASA Primary Scales}

We then investigated whether "threat," "challenge," or both would be significantly related to change in total D-dimer AUC. Again, a significance level of $p=.025$ was applied to account for multiple comparisons (i.e., $p=.05 / 2$ for "threat" and "challenge"). Table 2 shows that "challenge" ( $r=0.39$,

\section{A. Norepinephrine change}

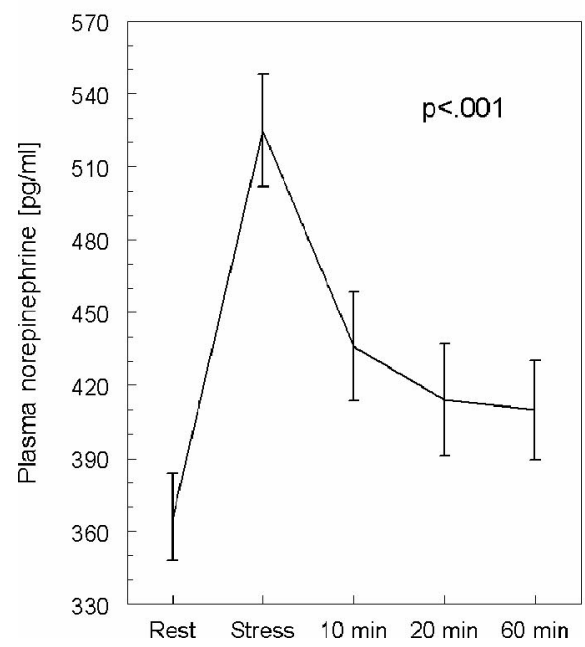

B. Epinephrine change

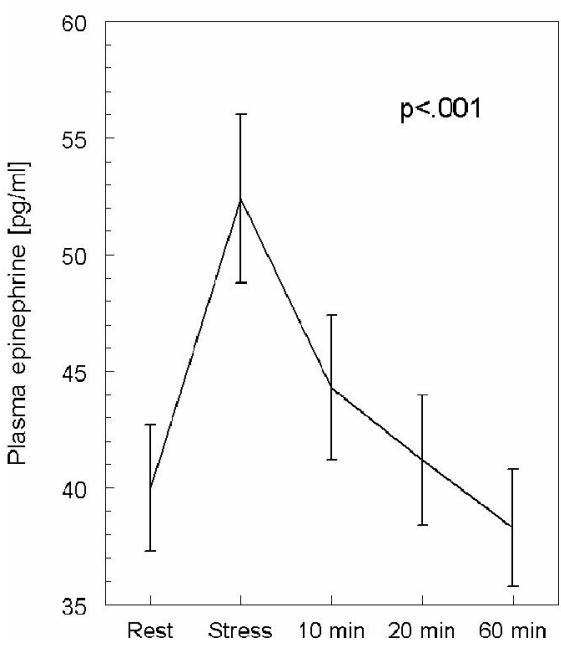

C. D-dimer change

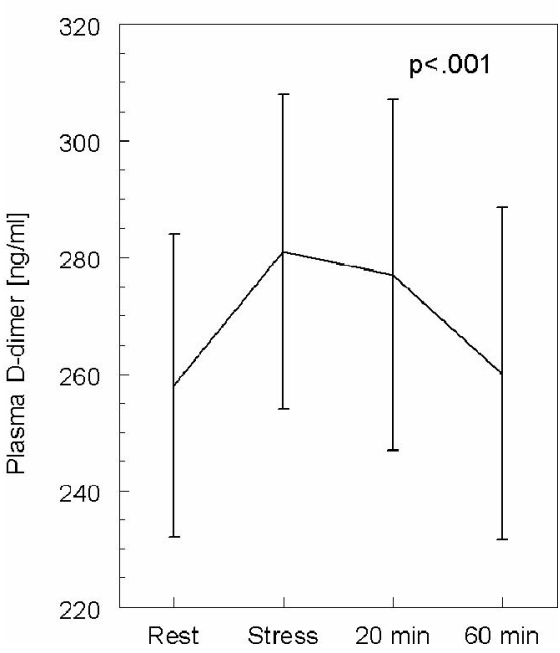

Figure 1. (A-C) The stressor elicited a significant increase in mean \pm standard error of mean of catecholamines and of D-dimer across all time points assessed (all $p$ values $<.001$ ). 
TABLE 2. Correlation Coefficients Between Primary Appraisal Secondary Appraisal Scales and D-dimer Measures

\begin{tabular}{|c|c|c|c|c|c|c|c|}
\hline & Threat & Challenge & Self-Concept & Control Expectancy & Primary Appraisal & Secondary Appraisal & Stress Index \\
\hline D-dimer at rest & 0.09 & 0.14 & -0.01 & 0.11 & 0.14 & 0.06 & 0.06 \\
\hline D-dimer total AUC & 0.23 & $0.39^{b}$ & -0.11 & -0.07 & $0.37^{a}$ & -0.12 & $0.30^{a}$ \\
\hline D-dimer stress change & 0.21 & $0.51^{c}$ & -0.09 & 0.07 & $0.41^{b}$ & -0.02 & $0.29^{a}$ \\
\hline D-dimer recovery AUC & 0.20 & $0.34^{a}$ & -0.11 & -0.06 & $0.32^{a}$ & -0.11 & $0.27^{d}$ \\
\hline
\end{tabular}

Correlations are significant at ${ }^{a} p \leq .05,{ }^{b} p \leq .01$, and ${ }^{c} p \leq .001$; a trend toward statistical significance is marked as ${ }^{d} p \leq .10$. Sample sizes were $n=47$ for resting D-dimer levels and for D-dimer change scores from rest to immediately poststress, and $n=42$ for the two measures of D-dimer area under the curve (AUC).

A. "Stress Index" and D-dimer total AUC

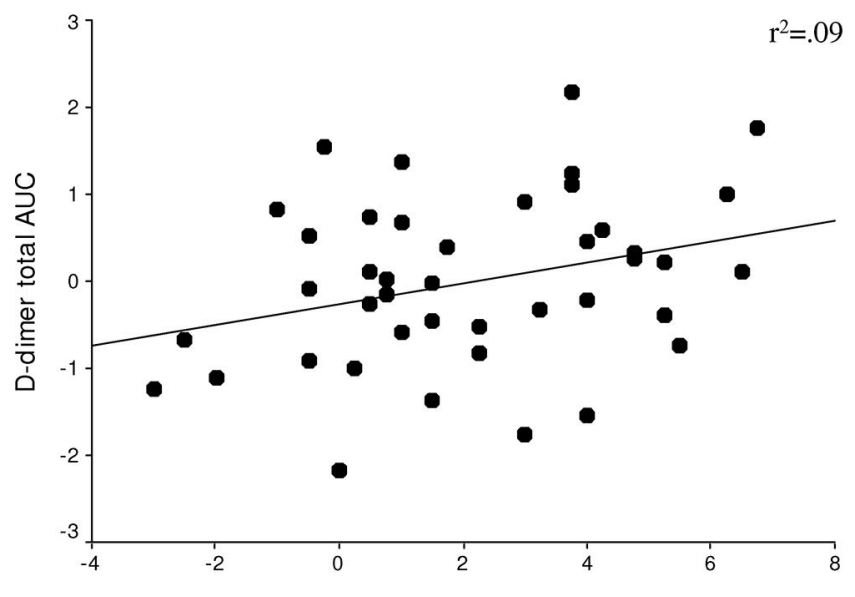

PASA Total Stress Index Score

C. "Challenge" and D-dimer stress change

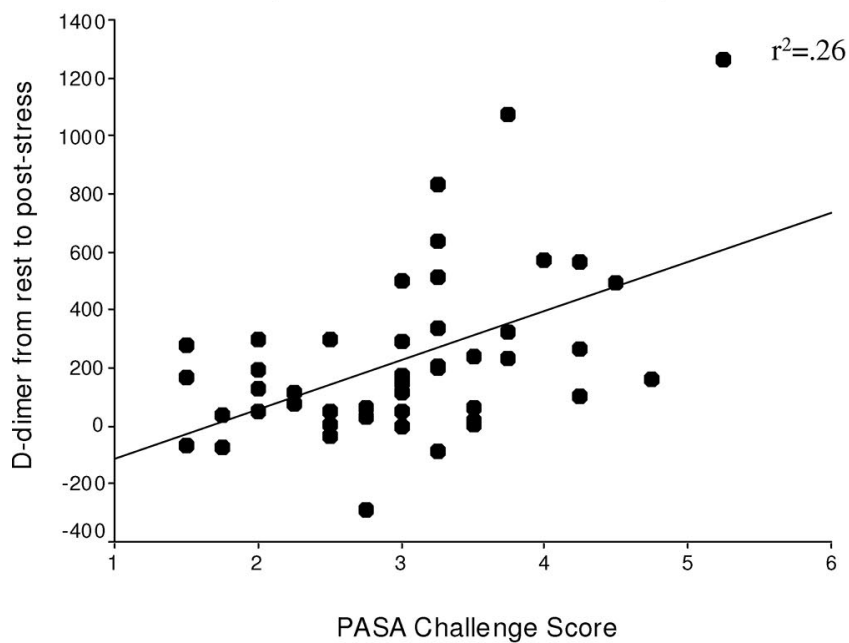

B. "Challenge" and D-dimer total AUC

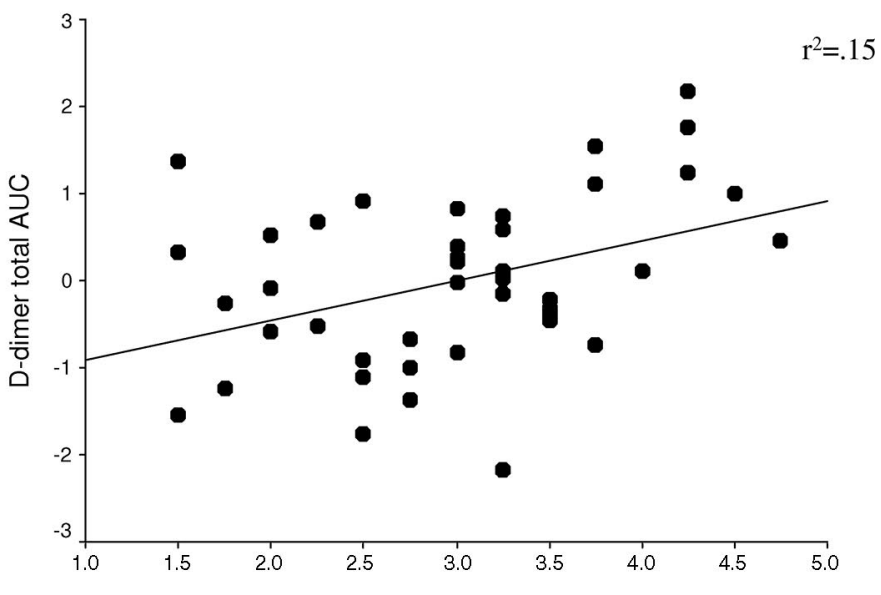

PASA Challenge Score

D. "Challenge" and D-dimer recovery

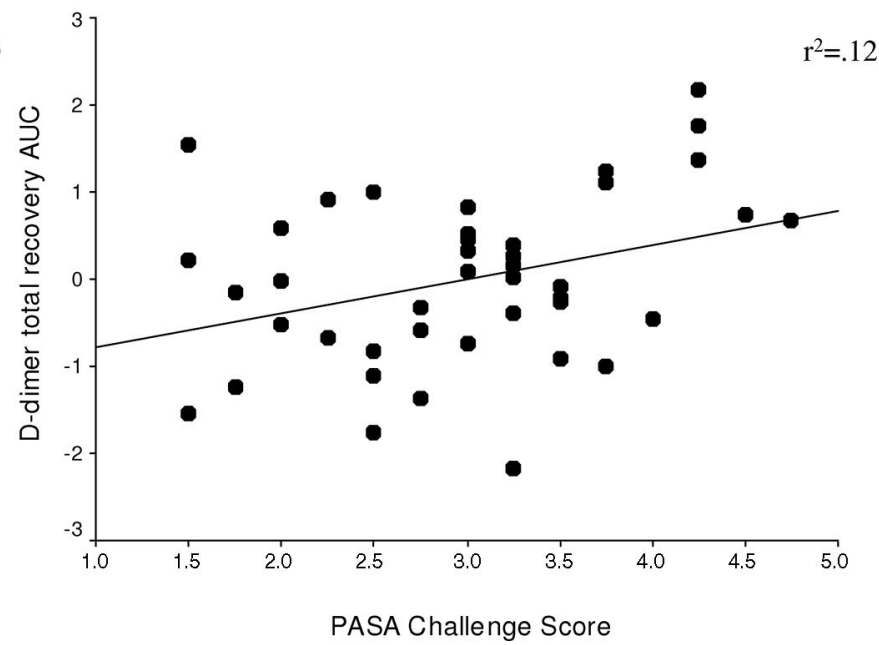

Figure 2. (A) Significant relationships between "total stress" and D-dimer total area under the curve (AUC) across all time points (omnibus test). (B-D) Significant relationships between "challenge" and D-dimer measures for the three time intervals. D-dimer values are expressed as arbitrary AUC units, which, moreover, were normalized for (A and B) D-dimer total AUC and (D) D-dimer recovery AUC.

$p=.010$; Fig. $2 \mathrm{~B})$ but not "threat" $(p=.15)$ of the primary appraisal scale was significantly associated with D-dimer total AUC. Nonetheless, the positive relationship between "threat" and D-dimer total AUC was in the predicted direction. Correlation coefficients between D-dimer total AUC and "threat" $(r=0.23)$ and "challenge" $(r=0.39)$, respectively, were not significantly different $(p=.27)$. This suggests that "challenge" and "threat" had a similarly strong effect on total integrated D-dimer formation but that the relationship between "threat" and D-dimer total AUC did not reach statistical significance because of limited statistical power.

Post hoc analysis showed that "challenge" but not "threat" correlated significantly with both D-dimer stress change ( $r=$ $0.51, p<.001$; Fig. $2 \mathrm{C})$ and D-dimer recovery AUC $(r=$ $0.34, p=.029$; Fig. 2D). Correlation coefficients between D-dimer stress change and "threat" $(r=0.21)$ and "challenge" 
TABLE 3. Hierarchical Regression Analyses for Primary Appraisal and D-dimer Measures

\begin{tabular}{|c|c|c|c|c|c|}
\hline Variables Entered & $\begin{array}{c}\text { Partial } \\
\text { Correlations }\end{array}$ & $\begin{array}{c}\text { Standardized } \\
\beta \text {-coefficient }\end{array}$ & $t$ & $p$ & $R^{2}$ Change \\
\hline \multicolumn{6}{|l|}{ D-dimer total AUC $(n=42)$} \\
\hline Age & 0.29 & 0.35 & 1.83 & .076 & 0.173 \\
\hline MBP & -0.01 & -0.01 & -0.07 & .948 & 0.010 \\
\hline BMI & -0.17 & -0.18 & -1.06 & .297 & 0.066 \\
\hline NEPI & 0.17 & 0.17 & 1.01 & .318 & 0.014 \\
\hline PA score & 0.21 & 0.20 & 1.29 & .205 & 0.033 \\
\hline \multicolumn{6}{|c|}{ D-dimer stress change $(n=47)$} \\
\hline Age & 0.14 & 0.15 & 0.90 & .374 & 0.083 \\
\hline MBP & -0.21 & -0.19 & -1.39 & .171 & 0.071 \\
\hline BMI & -0.07 & -0.07 & -0.42 & .677 & 0.035 \\
\hline NEPI & 0.30 & 0.28 & 2.03 & .049 & 0.037 \\
\hline PA score & 0.36 & 0.37 & 2.43 & .019 & 0.098 \\
\hline \multicolumn{6}{|c|}{ D-dimer recovery AUC $(n=42)$} \\
\hline Age & 0.26 & 0.32 & 1.61 & .117 & 0.159 \\
\hline MBP & 0.02 & 0.01 & 0.09 & .928 & 0.006 \\
\hline BMI & -0.14 & -0.14 & -0.82 & .419 & 0.050 \\
\hline NEPI & 0.20 & 0.20 & 1.21 & .235 & 0.025 \\
\hline PA score & 0.16 & 0.16 & 0.99 & .330 & 0.020 \\
\hline
\end{tabular}

$\mathrm{AUC}=$ area under the curve $\mathrm{BMI}=$ body mass index $\mathrm{MBP}=$ mean blood pressure NEPI $=$ norepinephrine change for the respective interval; $\mathrm{PA}=$ primary appraisal.

$(r=0.51)$, respectively, were significantly different $(p=$ $.020)$, suggesting that "challenge" was the "driving force" behind D-dimer formation from rest to immediately poststress. In contrast, correlation coefficients between D-dimer recovery AUC and "threat" $(r=0.20)$ and "challenge" $(r=0.34)$, respectively, did not differ $(p=.34)$, suggesting that the two scales contributed with similar strength to D-dimer recovery from stress.

\section{Covariate Analysis}

We calculated hierarchical regression equations in which age, MBP, BMI, NEPI activity, and "primary appraisal" were entered and regressed on D-dimer measures in this sequence (Table 3). All three models were significant with most of the variance explained for $\mathrm{D}$-dimer stress change $\left(\mathrm{R}^{2}=0.324\right.$, $\left.\mathrm{F}_{5,41}=3.9, p=.005\right)$, somewhat less variance explained for $\mathrm{D}$-dimer total AUC $\left(\mathrm{R}^{2}=0.296, \mathrm{~F}_{5,36}=3.0, p=.022\right)$, and the least variance explained for D-dimer recovery AUC $\left(\mathrm{R}^{2}=\right.$ $0.260, \mathrm{~F}_{5,36}=2.5, p=.046$ ).

"Primary appraisal" was an independent predictor of Ddimer stress change, explaining almost $10 \%$ of the variance (Table 3). The regression coefficient (b) indicated that for one-unit increase in "primary appraisal," mean \pm standard error of mean increase in D-dimer from rest to immediately poststress was $12.8 \pm 5.3 \mathrm{ng} / \mathrm{mL}$. Moreover, NEPI and Ddimer stress change were also independently associated, suggesting that the more NEPI was released, the more D-dimer was generated. Neither "primary appraisal" nor age, BMI, MBP, and NEPI activity measures emerged as significant predictors of D-dimer total AUC and D-dimer recovery AUC.

In post hoc analyses, "threat" did not emerge as an independent predictor of D-dimer measures in any model ( $p$ values $>$.27). In contrast, "challenge" was the only significant pre- dictor of $\mathrm{D}$-dimer stress change $\left(\mathrm{R}^{2}=0.138, \beta=0.40, p=\right.$ $.005)$. For a one-unit increase in "challenge," $\mathrm{D}$-dimer increased by $13.3 \pm 4.4 \mathrm{ng} / \mathrm{mL}$. In addition, "challenge" showed a trend toward statistical significance as a predictor of total D-dimer AUC $\left(\mathrm{R}^{2}=0.059, \beta=0.26, p=.085\right)$ but did not predict D-dimer recovery AUC $(p=.20)$.

\section{DISCUSSION}

We verified stress responsiveness of D-dimer previously found in study samples different from that investigated in the present study $(18,21)$. Most importantly, we confirmed our primary hypothesis, showing that anticipatory cognitive appraisal related to acute psychosocial stress was directly associated with stress-induced increase in this hypercoagulability marker. A more detailed analysis revealed that cognitive appraisal of the stressor was more strongly related to the amount of D-dimer increase from rest to immediately poststress than to the integrated change in D-dimer levels during recovery.

In line with our previous findings on the association between stress-induced cortisol secretion and "primary appraisal" (i.e., combined "threat" and "challenge") (17), we found here that of the secondary appraisal scales, "primary appraisal" but not "secondary appraisal" significantly correlated with D-dimer change across all three time intervals assessed. This observation suggests that perceived competence and control over the stressor had little effect on procoagulant reactivity. In other words, it was not primarily the subjects' perception as to whether their resources would be sufficient to cope with the task, but rather their interpretation of stressor characteristics as threatening and challenging that related to the procoagulant stress response. The strength of the associations of "threat" and "challenge," respectively, with individual D-dimer measures showed that "challenge" was 
more strongly related to D-dimer generation from rest to immediately poststress than "threat." In contrast, anticipated threat and challenge contributed with similar strength to total D-dimer formation and recovery of D-dimer change from stress, respectively. Our findings therefore add to an emerging array of factors that may modulate the acute procoagulant stress response $(10-12)$. In addition, our data suggest that the use of the transactional stress model for identifying psychophysiological mechanisms pertinent to cardiovascular diseases might depend on whether facets of "primary appraisal" or of "secondary appraisal" are studied.

Of the covariates, NEPI activity independently predicted D-dimer stress change, confirming previous research on catecholamine effects on the hypercoagulable state (27), whereas age, MBP, and BMI were not independent predictors of any D-dimer measure. Similarly, NEPI activity did not predict total D-dimer AUC and D-dimer recovery AUC. Despite the fact that these covariates did not emerge as independent predictors, the significant relationship between "primary appraisal" and D-dimer total AUC and D-dimer recovery AUC became nonsignificant when age, MBP, BMI, and NEPI activity were controlled for. Although this could reflect a decrease in power, a more parsimonious interpretation would be that effects of "primary appraisal" on these D-dimer measures disappeared as a result of covariates. However, and as we hypothesized, even when these common covariates of hemostatic function were controlled for, "primary appraisal," and particularly its subscale "challenge" but not "threat," remained independent predictors of the amount of fibrin formed between rest and immediately poststress. In addition, "challenge" reached borderline significance as an independent predictor of total D-dimer AUC. However, as a result of our sample size, we feel that it would be premature to conclude with any certainty that physiological changes accompanying the perception of stress as challenging really do elicit fibrin formation more readily than the perceived threat related to the stressor.

Besides the sample size and the disparity of our sample in terms of covariates, which render the data somewhat difficult to interpret, four additional limitations of our study should be mentioned. First, our device to measure BP yielded relatively high BP values compared with those obtained by sphygmomanometry (data not shown). However, we feel that this methodological caveat did not bias the significance of the BP response to stress. Second, we investigated nonsmoking men in reasonable mental and physical health, some of whom had cardiovascular risk factors in terms of overweight and mild hypertension, but we excluded subjects with manifest CAD. Our findings are therefore not readily transferable to patients with CAD, who are likely to be the population that manifests the greatest atherothrombotic risk with stress (4-9). Although BP and BMI were not correlated with D-dimer change in our subjects, we do not know whether these cardiovascular risk factors would increase procoagulant reactivity against a background of atherosclerotic vessels, which express hampered endothelial anticoagulant function (39). Third, we did not investigate women, and it therefore remains unclear whether women show a similarly extensive procoagulant stress response to that of men when evaluating the TSST as threatening and challenging. This is even more uncertain in light of previous research suggesting that transactional stress processes could be involved in the development of negative affect in women, who encounter life stress more than men (40). Fourth, anxiety and neuroticism showed no association with any D-dimer measure (data not shown); however, we cannot discount the possibility that some other co-occurring psychosocial factor previously shown to affect the D-dimer stress response $(10-12,18)$ contributed to our findings.

Our findings may have clinical implications in that they suggest that cognitive training of subjects with high anticipatory "primary appraisal" could provide a psychophysiological benefit. If cognitive training is directed at helping subjects to perceive a stressor as less threatening or challenging, than is realistic in a given context, the procoagulant stress response might be less exaggerated. For instance, we have previously demonstrated that a cognitive-behavioral stress inoculation program led to lower stress appraisal, which was accompanied by a decrease in the hypothalamic-pituitary adrenal axis response to acute psychosocial stress $(41,42)$. The clinical importance of stress inoculation programs could grow tremendously if researchers succeed in demonstrating that stress management in its broadest sense alters physiological systems, in our case, hemostatic stress responses, which further downstream and prospectively alleviate cardiovascular risk (14).

In summary, our findings suggest that anticipatory cognitive appraisal of acute stress is associated with the procoagulant stress response in men. In particular, we observed that D-dimer was generated as more pronounced in subjects who perceived more threat and challenge in relation to the stressor. In part, this effect was independent of common correlates of coagulation activity. Future intervention studies need to show whether the procoagulant response to stress can be modified by teaching effective coping skills and whether the resulting attenuation of procoagulant reactivity will benefit patients with atherothrombotic diseases.

\section{REFERENCES}

1. Colman RW, Hirsh J, Marder VJ, Clowes AW, George JN, eds. Hemostasis and Thrombosis. Basic Principles and Clinical Practice, 4th ed. Philadelphia: Lippincott Williams \& Wilkins; 2001.

2. von Känel R, Mills PJ, Fainman C, Dimsdale JE. Effects of psychological stress and psychiatric disorders on blood coagulation and fibrinolysis: a biobehavioral pathway to coronary artery disease? Psychosom Med 2001; 63:531-44.

3. Cannon WB, Mendenhall WL. Factors affecting the coagulation time of blood. IV. The hastening of coagulation in pain and emotional excitement. Am J Physiol 1914;34:251-61.

4. Preckel D, von Känel R. Regulation of hemostasis by the sympathetic nervous system - any contribution to coronary artery disease? Heart Drug 2004; 4:123-30.

5. Strike PC, Magid K, Brydon L, Edwards S, McEwan JR, Steptoe A. Exaggerated platelet and hemodynamic reactivity to mental stress in men with coronary artery disease. Psychosom Med 2004;66:492-500.

6. von Känel R, Dimsdale JE, Ziegler MG, Mills PJ, Patterson TL, Lee SK, Grant I. Effect of acute psychological stress on the hypercoagulable state in subjects (spousal caregivers of patients with Alzheimer's disease) with 
coronary or cerebrovascular disease and/or systemic hypertension. Am J Cardiol 2001;87:1405-8.

7. Canevari A, Sacconi F, Zucchella M, Pacchiarini L, Soffiantino F, Grignani G. Antithrombin III biological activity and emotional stress in patients with coronary artery disease. Haematologica 1992;77:180-2.

8. Palermo A, Bertalero P, Pizza N, Amelotti R, Libretti A. Decreased fibrinolytic response to adrenergic stimulation in hypertensive patients. J Hypertens Suppl 1989;7:S162-3.

9. Mittleman MA, Maclure M, Sherwood JB, Mulry RP, Tofler GH, Jacobs SC, Friedman R, Benson H, Muller JE. Triggering of acute myocardial infarction onset by episodes of anger. Determinants of Myocardial Infarction Onset Study Investigators. Circulation 1995;92:1720-5.

10. von Känel R, Dimsdale JE, Patterson TL, Grant I. Acute procoagulant stress response as a dynamic measure of allostatic load in Alzheimer caregivers. Ann Behav Med 2003;26:42-8.

11. von Känel R, Dimsdale JE, Adler KA, Patterson TL, Mills PJ, Grant I. Effects of depressive symptoms and anxiety on hemostatic responses to acute mental stress and recovery in the elderly. Psychiatry Res 2004;126: 253-64.

12. Aschbacher K, Patterson TL, von Känel R, Dimsdale JE, Mills PJ, Adler KA, Ancoli- Israel S, Grant I. Coping processes and hemostatic reactivity to acute stress in dementia caregivers. Psychosom Med 2005;67:964-71.

13. Skinner EA, Edge K, Altman J, Sherwood H. Searching for the structure of coping: a review and critique of category systems for classifying ways of coping. Psychol Bull 2003;129:216-69.

14. Linden W. Stress Management. From Basic Science to Better Practice. Thousand Oaks, CA: Sage Publications; 2005.

15. Lazarus RS, Folkman S. Stress, Appraisal, and Coping. New York: Springer Publishing Co; 1984.

16. Park CL. Stress-related growth and thriving through coping: the roles of personality and cognitive processes. J Soc Issues 1998;54:267-77.

17. Gaab J, Rohleder N, Nater UM, Ehlert U. Psychological determinants of the cortisol stress response: the role of anticipatory cognitive appraisal. Psychoneuroendocrinology 2005;30:599-610.

18. von Känel R, Dimsdale JE. Fibrin D-dimer: a marker of psychosocial distress and its implications for research in stress-related coronary artery disease. Clin Cardiol 2003;26:164-8.

19. Lip GY, Lowe GD. Fibrin D-dimer: a useful marker of thrombogenesis? Clin Sci 1995;89:205-14.

20. Chandler WL, Velan T. Plasmin generation and D-dimer formation during cardiopulmonary bypass. Blood Coagul Fibrinolysis 2004;15: 583-91.

21. von Känel R, Preckel D, Zgraggen L, Mischler K, Kudielka BM, Haeberli A, Fischer JE. The effect of natural habituation on coagulation responses to acute mental stress and recovery in men. Thromb Haemost 2004;92: $1327-35$.

22. Zierler BK. Ultrasonography and diagnosis of venous thromboembolism. Circulation 2004;109(suppl 1):I9-14.

23. Danesh J, Whincup P, Walker M, Lennon L, Thomson A, Appelby P, Rumley A, Lowe GD. Fibrin D-dimer and coronary heart disease: prospective study and meta-analysis. Circulation 2001;103:2323-7.

24. Hughes R, Thomson K, Hopkins R, Weatherall M, Wiltshire C, Wilsher $\mathrm{M}$, Beasley R. Determinants of plasma D-dimer levels in a traveling population. J Thromb Haemost 2005;3:2445-8.

25. Yarnell JW, Sweetnam PM, Rumley A, Lowe GD. Lifestyle factors and coagulation activation markers: the Caerphilly Study. Blood Coagul Fibrinolysis 2001;12:721-8.

26. Catena C, Novello M, Lapenna R, Baroselli S, Colussi G, Nadalini E,
Favret G, Cavarape A, Soardo G, Sechi LA. New risk factors for atherosclerosis in hypertension: focus on the prothrombotic state and lipoprotein(a). J Hypertens 2005;23:1617-31.

27. von Känel R, Dimsdale JE. Effects of sympathetic activation by adrenergic infusions on hemostasis in vivo. Eur J Haematol 2000;65:357-69.

28. McEwen BS. Protective and damaging effects of stress mediators. N Engl J Med 1998;338:171-9.

29. Larsen LF, Andersen HR, Hansen AB, Andersen O. Variation in risk indicators of cardiovascular disease during the menstrual cycle: an investigation of within-subject variations in glutathione peroxidase, haemostatic variables, lipids and lipoproteins in healthy young women. Scand J Clin Lab Invest 1996;56:241-9.

30. Jern C, Manhem K, Eriksson E, Tengborn L, Risberg B, Jern S. Hemostatic responses to mental stress during the menstrual cycle. Thromb Haemost 1991;66:614-8.

31. von Känel R, Kudielka BM, Abd-el-Razik A, Gander ML, Frey K, Fischer JE. Relationship between overnight neuroendocrine activity and morning haemostasis in working men. Clin Sci 2004;107:89-95.

32. Kirschbaum C, Pirke KM, Hellhammer DH. The 'Trier Social Stress Test' $-\mathrm{a}$ tool for investigating psychobiological stress responses in a laboratory setting. Neuropsychobiology 1993;28:76-81.

33. Kudielka BM, Schommer NC, Hellhammer DH, Kirschbaum C. Acute HPA axis responses, heart rate, and mood changes to psychosocial stress (TSST) in humans at different times of day. Psychoneuroendocrinology 2004;29:983-92.

34. Belani KG, Ozaki M, Hynson J, Hartmann T, Reyford H, Martino JM, Poliac M, Miller R. A new noninvasive method to measure blood pressure: results of a multicenter trial. Anesthesiology 1999;91:686-92.

35. Sostaric M, Hartmann T, Zimpfer M, Poliac M, Belani K. Comparison of the Vasotrac BP monitor with the arm cuff methods. Anesthesiology 2000;98:A-348.

36. Ehrenreich H, Schuck J, Stender N, Pilz J, Gefeller O, Schilling L, Poser W, Kaw S. Endocrine and hemodynamic effects of stress versus systemic $\mathrm{CRF}$ in alcoholics during early and medium term abstinence. Alcohol Clin Exp Res 1997;21:1285-93.

37. Pruessner JC, Kirschbaum C, Meinlschmid G, Hellhammer DH. Two formulas for computation of the area under the curve represent measures of total hormone concentration versus time-dependent change. Psychoneuroendocrinology 2003;28:916-31.

38. Babyak MA. What you see may not be what you get: a brief, nontechnical introduction to overfitting in regression-type models. Psychosom Med 2004; 66:411-21.

39. Cines DB, Pollak ES, Buck CA, Loscalzo J, Zimmerman GA, McEver RP, Pober JS, Wick TM, Konkle BA, Schwartz BS, Barnathan ES, McCrae KR, Hug BA, Schmidt AM, Stern DM. Endothelial cells in physiology and in the pathophysiology of vascular disorders. Blood 1998;91:3527-61.

40. Hankin BL, Abramson LY. Development of gender differences in depression: an elaborated cognitive vulnerability-transactional stress theory. Psychol Bull 2001;127:773-96.

41. Gaab J, Blattler N, Menzi T, Pabst B, Stoyer S, Ehlert U. Randomized controlled evaluation of the effects of cognitive-behavioral stress management on cortisol responses to acute stress in healthy subjects. Psychoneuroendocrinology 2003;28:767-79.

42. Hammerfald K, Eberle C, Grau M, Kinsperger A, Zimmermann A, Ehlert U, Gaab J. Persistent effects of cognitive-behavioral stress management on cortisol responses to acute stress in healthy subjects - a randomized controlled trial. Psychoneuroendocrinology 2006;31:333-9. 